\title{
Detecting nonlocality of noisy multipartite states with the Clauser-Horne-Shimony-Holt inequality
}

\author{
Rafael Chaves, ${ }^{1}$ Antonio Acín, ${ }^{2,3}$ Leandro Aolita, ${ }^{4}$ and Daniel Cavalcanti ${ }^{2}$ \\ ${ }^{1}$ Institute for Physics, University of Freiburg, Rheinstrasse 10, D-79104 Freiburg, Germany \\ ${ }^{2}$ ICFO-Institut de Ciències Fotòniques, Mediterranean Technology Park, E-08860 Castelldefels (Barcelona), Spain \\ ${ }^{3}$ ICREA-Institucio Catalana de Recerca i Estudis Avançats, 08010 Barcelona, Spain \\ ${ }^{4}$ Dahlem Center for Complex Quantum Systems, Freie Universität Berlin, D-14195 Berlin, Germany \\ (Received 21 November 2013; published 14 April 2014; corrected 20 January 2015)
}

\begin{abstract}
The Clauser-Horne-Shimony-Holt inequality was originally proposed as a Bell inequality to detect nonlocality in bipartite systems. However, it can also be used to certify the nonlocality of multipartite quantum states. We apply this to study the nonlocality of multipartite Greenberger-Horne-Zeilinger (GHZ), $W$, and graph states under local decoherence processes. We derive lower bounds on the critical local-noise strength tolerated by the states before becoming local. In addition, for the whole noisy dynamics, we derive lower bounds on the corresponding nonlocal content for the three classes of states. All the bounds presented can be calculated efficiently and, in some cases, provide significantly tighter estimates than with any other known method. For example, they reveal that $N$-qubit GHZ states undergoing local dephasing are, for all $N$, nonlocal throughout all the dephasing dynamics.

DOI: 10.1103/PhysRevA.89.042106

PACS number(s): 03.65.Ud, 03.67.Mn
\end{abstract}

\section{INTRODUCTION}

Nonlocality refers to correlations between the measurement results of distant systems that cannot be explained by local hidden-variable (LHV) models [1,2]. The correlations consistent with a LHV model necessarily satisfy a set of linear constraints known as Bell inequalities [2], which can be experimentally tested. Thus, the violation of any Bell inequality reveals the presence of nonlocality. In addition, apart from a fundamental issue, the detection of nonlocal correlations is also of practical relevance. First, the violation of a Bell inequality is a device-independent entanglement witness, i.e., it allows one to certify entanglement in situations where the sources and measurements implemented are totally unknown [2,3]. Second, the efficacy at solving informationtheoretic tasks such as communication complexity problems [4], device-independent quantum key distribution [5-7], and randomness extraction [8,9] or amplification [10-12] relies on the presence of nonlocality. Experimentally friendly ways to extract nonlocal correlations from quantum states appears thus highly desirable.

The simplest way to do this, in the case of two parts with two dichotomic measurements each, is through the $\mathrm{CHSH}$ inequality [13],

$$
\mathrm{CHSH} \equiv\left\langle a_{0} b_{0}\right\rangle+\left\langle a_{0} b_{1}\right\rangle+\left\langle a_{1} b_{0}\right\rangle-\left\langle a_{1} b_{1}\right\rangle \leqslant 2,
$$

where $a_{x}= \pm 1$ and $b_{y}= \pm 1$ are the outcomes of measurement settings labeled by $x=\{0,1\}$ and $y=\{0,1\}$ for Alice and Bob, respectively, and $\left\langle a_{x} b_{y}\right\rangle=p(a=b \mid x y)-p(a \neq b \mid x y)$ stands for the statistical average of $a_{x} b_{y}$. In quantum mechanics these averages can be expressed by $\left\langle a_{x} b_{y}\right\rangle \equiv \operatorname{Tr}\left[\hat{A}_{x} \otimes\right.$ $\hat{B}_{y} \rho$ ], where $\hat{A}_{x}$ and $\hat{B}_{y}$ are Hermitian observables with eigenvalues \pm 1 and $\rho$ a quantum state. The CHSH inequality (and its symmetries) is the only relevant Bell inequality in the bipartite scenario with two dichotomic measurements [14], i.e., it can tightly capture all nonlocal correlations. Furthermore, for two-qubit quantum states, CHSH violation can be immediately checked via the necessary and sufficient condition found in [15].
In the multipartite scenario, however, the situation changes drastically. For instance, already for the modest case of three parts applying two dichotomic measurements each, there are 46 inequivalent classes of nontrivial and tight Bell inequalities [16]. In general, the efficiency in the characterization of nonlocality as the number of parts, measurements or outcomes increases becomes a major issue. In fact, deciding the compatibility of a given probability distribution with LHV models is known to be an NP-complete problem $[17,18]$.

In this paper, we study the nonlocality of genuinely multipartite $N$-qubit Greenberger-Horne-Zeilinger (GHZ), $W$, and graph states under local decoherence processes described by Pauli channels. We derive lower bounds on the critical local-noise strength tolerated by the states before becoming local, in a similar spirit as in [19]. In addition, for each noise strength, we derive lower bounds on the nonlocal content [20] of the correlations on the three classes of states. The bounds we derive are based on the CHSH violation of two out of the $N$ qubits conditioned on a measurement outcome of all other $N-2$ qubits [21], and can therefore be calculated efficiently. As a matter of fact, we show that in some cases, such as with GHZ states under transversal local dephasing (bit-flip noise), the bounds obtained are even $N$ independent. Furthermore, we show that the estimates given by these bounds are (in some cases exponentially) tighter than those given by any other known method.

In Sec. II we introduced the different classes of states, the noise models, and figures of merit for nonlocality to be used in this paper. In Sec. III we describe the general method that is applied in Sec. IV to derive, respectively, lower bounds on the critical noise strength and the nonlocal content. In Sec. V we present a summary of the results while some technical results about graph states Bell inequalities are relegated to the Appendix.

\section{STATES, NOISE MODELS, AND FIGURES OF MERIT}

In this section, we introduce basic notation, define the states studied, the noise channels considered and the figures of merit we use to assess the nonlocality of noisy states. 


\section{A. States under scrutiny}

We consider three paradigmatic families of genuinely multipartite $N$-qubit quantum states:

(1) GHZ states [22],

$$
\left|\mathrm{GHZ}_{N}\right\rangle \doteq \frac{1}{\sqrt{2}}\left(|0\rangle^{\otimes N}+|1\rangle^{\otimes N}\right) ;
$$

(2) $W$ states [23],

$$
\left|W_{N}\right\rangle \doteq \frac{1}{\sqrt{N}}(|0 \ldots 01\rangle+|0 \ldots 10\rangle+\cdots+|10 \ldots 0\rangle) \text {; }
$$

(3) Graph states $[24,25]$. A graph state $\left|G_{\mathbf{0}}\right\rangle$ is associated with an $N$-vertex mathematical graph $G$, whose geometry is determined by a set $\mathcal{E}$ of edges $\{i, j\}$ indicating which vertices $i$ and $j$ are connected, for $1 \leqslant i, j \leqslant N$. More precisely,

$$
\left|G_{\mathbf{0}}\right\rangle \doteq \mathrm{CZ}_{\mathcal{E}}|+\rangle^{\otimes N}
$$

being $|+\rangle \doteq(|0\rangle+|1\rangle) / \sqrt{2}$ and $\mathrm{CZ}_{\mathcal{E}} \doteq \prod_{\{i, j\} \in \mathcal{E}} \mathrm{CZ}_{i, j}$, where $\mathrm{CZ}_{i, j} \doteq e^{\left(Z_{i}-\mathbb{1}_{i}\right) \otimes\left(Z_{j}-\mathbb{1}_{j}\right) / 4} \otimes \mathbb{1}_{\overline{i, j}}$ is the maximally entangling controlled-Z gate nontrivially acting on qubits $i$ and $j$, with $Z_{i}$ and $Z_{j}$ the third Pauli operators on qubits $i$ and $j$, respectively, and $\mathbb{1}_{i}, \mathbb{1}_{j}$, and $\mathbb{1}_{\overline{i, j}}$ the identity operators on qubits $i, j$, and all but $i$ and $j$, respectively, for any $1 \leqslant i, j \leqslant N$.

\section{B. Decoherence models}

As noise models we consider local Pauli channels of the form

$$
\Lambda\left(\rho_{0}\right) \doteq \sum_{i=0}^{3} p_{i} \sigma_{i} \rho_{0} \sigma_{i}
$$

Here, $\rho_{0}$ is any initial state and $\Lambda$ is a single-qubit Pauli channel. $\sigma_{0} \doteq \mathbb{1}$, and $\sigma_{1} \doteq X, \sigma_{2} \doteq Y$, and $\sigma_{3} \doteq Z$ refer to the usual Pauli operators. The coefficients $p_{i}$ satisfy the relation$\operatorname{ship} p_{0}=(1-p / 2), p_{1}=\alpha_{1} p / 2, p_{2}=\alpha_{2} p / 2, p_{3}=\alpha_{3} p / 2$, with $\alpha_{1}+\alpha_{2}+\alpha_{3}=1$ so that the total noise strength $0 \leqslant$ $p \leqslant 1$ is distributed along the three Bloch-sphere directions according to $\alpha_{1}, \alpha_{2}$, and $\alpha_{3}$. For example, the case $\alpha_{1}=\alpha_{2}=0$ describes dephasing along the direction $z$ of the Bloch sphere (also known as phase-flip channel). Analogously, $\alpha_{2}=\alpha_{3}=0$ describes dephasing along the transversal direction $x$ (bit-flip channel). We consider joint evolutions given by independent and identical channels on all qubits:

$$
\rho_{p}=\Lambda^{\otimes N}\left(\rho_{0}\right)
$$

\section{Figures of merit}

To assess the nonlocal correlations in quantum states, we focus mainly in two quantities. The first one is the critical noise strength $p_{c}$ beyond which no nonlocality can be extracted [19]. We refer to $p_{c}$ as the noise threshold and in the following we compute a lower bound to it.

The second one is the amount of nonlocality for each noise strength $p$, which we quantify through the EPR2 decomposition [20]. Any joint-probability distribution $P$, characterizing the correlations of some Bell experiment, can be decomposed into a convex mixture of a local part $P_{L}$ and a general nonlocal (no-signaling) part $P_{\mathrm{NL}}$ as $P=\left(1-p_{\mathrm{NL}}\right) P_{L}+p_{\mathrm{NL}} P_{\mathrm{NL}}$, with $0 \leqslant p_{\mathrm{NL}} \leqslant 1$. The minimal nonlocal weight over all such decompositions,

$$
\tilde{p}_{\mathrm{NL}} \doteq \min _{P_{L}, P_{\mathrm{NL}}} p_{\mathrm{NL}}
$$

defines the nonlocal content of $P$, which provides a natural quantifier of the nonlocality in $P$. In turn, we define the nonlocal content of a quantum state as the maximum nonlocal content of correlations over all possible Bell experiments with the state.

It turns out that the violation of any Bell inequality yields a nontrivial lower bound to $\tilde{p}_{\mathrm{NL}}$ [26]. For any (linear) Bell inequality $\mathcal{I} \leqslant \mathcal{I}^{L}$, with $\mathcal{I}^{L}$ the local bound, it is

$$
\tilde{p}_{\mathrm{NL}} \geqslant \frac{\mathcal{I}(P)-\mathcal{I}^{L}}{\mathcal{I}^{N L}-\mathcal{I}^{L}},
$$

where $\mathcal{I}^{\mathrm{NL}}$ is the maximum Bell value $\mathcal{I}$ over all arbitrary nonsignaling correlations.

\section{THE METHOD}

We will consider a scenario where $N$ parties share a multipartite state and perform local measurements on it. Two of the parties apply two dichotomic measurements, labeled again by $x=\{0,1\}$ and $y=\{0,1\}$, with possible outcomes $a_{x}= \pm 1$ and $b_{y}= \pm 1$, respectively. The other $N-2$ parties apply only one dichotomic measurement each. We will denote the outcomes of these $N-2$ measurements by an $N-2$ component vector $\mathbf{c}=( \pm 1, \ldots, \pm 1)$. The Bell inequality that we will consider is given by (see Appendix B of Ref. [27])

$$
\mathrm{CHSH}_{\mathbf{c}} \equiv\left\langle a_{0} b_{0}\right\rangle_{\mathbf{c}}+\left\langle a_{0} b_{1}\right\rangle_{\mathbf{c}}+\left\langle a_{1} b_{0}\right\rangle_{\mathbf{c}}-\left\langle a_{1} b_{1}\right\rangle_{\mathbf{c}}-2 p(\mathbf{c}) \leqslant 0,
$$

where $\quad\left\langle a_{x} b_{y}\right\rangle_{\mathbf{c}}=[p(a=b \mid x, y, \mathbf{c})-p(a \neq b \mid x, y, \mathbf{c})] p(\mathbf{c})$. Notice that this inequality is simply the CHSH inequality calculated with the conditional probability distribution for the two parties given that the other $N-2$ parties get the particular outcome c.

Proof of the validity of Bell inequality (9). We need to show that all the local deterministic probability distributions, i.e., those assigning definite outcomes for each measurement, satisfy it. For the local deterministic distributions for which $p(\mathbf{c})=1$, inequality (9) becomes the standard CHSH inequality (1), while for the local deterministic strategies such that $p(\mathbf{c})=0$ it simply reads $0 \leqslant 0$.

Thus, in order to detect nonlocality in a given $N$-partite state $\rho$ through the inequality (9) we need to find appropriate local measurements on $N-2$ parts that project the remaining two parts into a bipartite state violating the $\mathrm{CHSH}$ inequality [21]. At this point it is worth emphasizing that the conditioning (or postselection) used in the present Bell test does not open any loophole. The reason is that it is done only on the outcomes of the $N-2$ parts which are spacelike separated from the two parties involved in the CHSH test. In this way, it could simply be seen as a heralded preparation of a nonlocal state by $N-2$ parties. Moreover, this method has already proven very useful in other contexts. For instance, it has been applied to prove that every multipartite pure entangled state is nonlocal [21], and to demonstrate superactivation of nonlocality in quantum networks [27-29]. 
Here, given projective measurements on the first $N-2$ qubits, we will test the nonlocality of the resulting two-qubit state through the necessary and sufficient condition for the CHSH violation discovered in Ref. [15]: The maximum CHSH value achievable by an arbitrary two-qubit state $\rho$ is

$$
\mathrm{CHSH}=2 \mathcal{M}_{\mathrm{CHSH}}(\rho)=2 \sqrt{t_{11}^{2}+t_{22}^{2}},
$$

being $t_{11}^{2}$ and $t_{22}^{2}$ the two largest eigenvalues of $\mathcal{T}^{\dagger} \mathcal{T}$, with $\mathcal{T}_{i, j}=\operatorname{tr}\left[\left(\sigma_{\mathrm{i}} \otimes \sigma_{\mathrm{j}}\right) \rho\right]$, where $\sigma_{i} \otimes \sigma_{j}$ refers to the product of the $i$ th and $j$ th Pauli operators on the two remaining qubits, for $1 \leqslant i, j \leqslant 3$. So, $\rho$ violates the CHSH inequality if, and only if, $\mathcal{M}_{\mathrm{CHSH}}(\rho)>1$.

As long as the probability $p(\mathbf{c})$ is greater than zero, its exact value does not affect the critical noise thresholds. Note, however, that using inequality (9) the lower bound for the nonlocal content will unavoidably depend on $p(\mathbf{c})$, namely,

$$
\tilde{p}_{\mathrm{NL}} \geqslant \frac{[\operatorname{CHSH}(\rho)-2] p(\mathbf{c})}{2} .
$$

For the states we consider in this paper, $p(\mathbf{c})$ will typically decay exponentially with the number of qubits $N$, also leading to an exponentially decaying lower bound. In order to circumvent that and still get non trivial lower bounds for the nonlocal content we proceed as follows.

For all the states we consider (with the exception of the $W$ state considered in Sec. IV B) all possible $2^{N-2}$ measurement outcomes lead to only two possible projected two-qubit states that, furthermore, are equivalent up to local unitaries. Let us call these projections events 1 and 2, the two respective projected states by $\rho_{1}$ and $\rho_{2}$, and $p(1)$ and $p(2)=1-p(1)$ the probabilities of events 1 and 2 .

We can then define Bell inequalities, similar to (9), to events 1 and 2 as

$$
\mathrm{CHSH}_{1} \equiv\left\langle a_{0} b_{0}\right\rangle_{1}+\left\langle a_{0} b_{1}\right\rangle_{1}+\left\langle a_{1} b_{0}\right\rangle_{1}-\left\langle a_{1} b_{1}\right\rangle_{1}-2 p(1) \leqslant 0,
$$

and

$$
\mathrm{CHSH}_{2} \equiv\left\langle a_{0} b_{0}\right\rangle_{2}+\left\langle a_{0} b_{1}\right\rangle_{2}-\left\langle a_{1} b_{0}\right\rangle_{2}+\left\langle a_{1} b_{1}\right\rangle_{2}-2 p(2) \leqslant 0,
$$

with $\left\langle a_{x} b_{y}\right\rangle_{i}=[p(a=b \mid x, y, i)-p(a \neq b \mid x, y, i)] p_{i}$. Finally we use these inequalities to define the following one:

$$
\mathrm{CHSH}_{1}+\mathrm{CHSH}_{2} \leqslant 0 \text {. }
$$

For most of the states we will consider here, we can find measurements $A_{0}, A_{1}, B_{0}, B_{1}$ that will lead to $p(1)=p(2)$ and $\mathrm{CHSH}_{1}=\mathrm{CHSH}_{2}$. This, in turn, will imply that the lower bound for the nonlocal content will be independent of the projection probabilities and simply given by $\tilde{p}_{\mathrm{NL}} \geqslant$ $\mathcal{M}_{\text {CHSH }}(\rho)-1$.

Finally notice that different Bell inequalities, conditioned on outputs of $N-2$ parties, could be similarly used. However, in the case that the two parties testing the Bell inequality have two binary inputs it is sufficient to consider the $\mathrm{CHSH}$ inequality.

\section{NONLOCALITY THRESHOLD AND NONLOCAL CONTENT OF NOISY STATES}

In this section we show how the multipartite $\mathrm{CHSH}$ method can be used to calculate the critical noise strength tolerated by the noisy state before becoming local. We also compute, for the entire noisy dynamics, lower bounds for the nonlocal content of the states. These lower bounds can be significantly better than the ones obtained via known multipartite inequalities.

\section{A. Noisy GHZ state}

We begin considering GHZ states. In particular, for parallel dephasing, we show that GHZ states of any number of qubits are nonlocal throughout all the noisy dynamics, a result that cannot be achieved by any other known multipartite inequality consisting exclusively of full correlators.

\section{Parallel dephasing}

We consider first the detection of nonlocality for the GHZ state (2) undergoing independent dephasing along the $Z$ direction. The resulting noisy GHZ state $\rho_{N}^{z}$ can be expressed as $[30,31]$

$\rho_{N}^{z}=(1-p)^{N}\left|\mathrm{GHZ}_{N}\right\rangle\left\langle\mathrm{GHZ}_{N}\right|+\left(1-(1-p)^{N}\right) \tilde{\varrho}_{N}^{z}$,

with $\tilde{\rho}_{N}^{z}=\left(|0\rangle\left\langle\left. 0\right|^{\otimes N}+\mid 1\right\rangle\left\langle\left. 1\right|^{\otimes N}\right) / 2\right.$.

We compare the multipartite CHSH method with the Werner-Wolf-Weinfurter-Zukowski-Brukner (WWWZB) inequalities [32-34]. These encompass all the $2^{2^{N}}$ tight, linear, full-correlator Bell inequalities in the $N$-partite scenario where each party makes two dichotomic measurements. In particular, of special relevance here is the Mermin-Klyshko (MK) inequality [35-37], which is a particular case of the WWWZB family. The MK inequality is the two-setting correlator Bell inequality with the largest violation in quantum theory [32], with an exponential maximal violation $2^{(N-1) / 2}$ (the local bound of the MK inequality is given by 1 ), achieved with the GHZ state for $X$ and $Y$ measurements.

The maximal MK violation for $\rho_{N}^{z}$ can be straightforwardly calculated [38] for the case of $N$ odd, to which we restrict for simplicity of notation. It is given by $2^{(N-1) / 2}(1-p)^{N}$ and is also attained with $X$ and $Y$ measurements. This yields in turn the noise threshold $p_{c}^{z}=1-1 / \sqrt{2^{(N-1) / N}}$, which is tighter than that given by any other known multipartite inequality consisting exclusively of full correlators.

We next show that the CHSH method renders $p_{c}^{z}=1$ for all $N$. Consider local $X$ measurements on the first $N-2$ qubits of (15) (Numerical optimization up to $N=5$ shows that these measurements are optimal, that is, they maximize the CHSH violation of the remaining two-qubit state. See [39] for further details). We consider explicitly the situation where all $N-2$ parties obtain the eigenvalue 1 , corresponding to the eigenstate $|+\rangle$. However, for any other outcome the treatment would be equivalent, except for a local-unitary relabeling of the projected states. This local-unitary equivalence will be explicitly used later on in order to derive lower bounds to the nonlocal content. The projected two-qubit state conditioned on the $N-2$ measurement outcomes obtained is

$$
\rho_{2}^{z}=(1-p)^{N}\left|\mathrm{GHZ}_{2}\right\rangle\left\langle\mathrm{GHZ}_{2}\right|+\left(1-(1-p)^{N}\right) \tilde{\rho}_{2}^{z} .
$$


Computing (10) for this state gives $\mathcal{M}_{\mathrm{CHSH}}^{z}=$ $\sqrt{1+(1-p)^{2 N}}$, which is greater than one for all $p<1$. Thus, GHZ states of arbitrary $N$ subject to independent parallel dephasing are nonlocal for any amount of dephasing $p<1$. We stress that such a high noise threshold cannot be detected by any other known multipartite inequality consisting exclusively of full correlators.

Interestingly, for $N=3$ this result can be made even stronger, since the CHSH method is able to detect the nonlocality in a region where any full-correlator inequality would fail. For the state (15) and $N$ odd it is not difficult to see that only the components of the projective measurement lying in the equatorial plane give a non-null contribution for full correlators. For example, for $N=3$ and the observable $O=\frac{(X+Z)}{\sqrt{2}} \otimes X \otimes X$ we have that $\operatorname{tr}\left(\mathrm{O} \rho_{\mathrm{N}}^{\mathrm{Z}}\right)=\operatorname{tr}((\mathrm{X} \otimes \mathrm{X} \otimes \mathrm{X})$ $\left.\left((1 / \sqrt{2}) \rho_{\mathrm{N}}^{\mathrm{Z}}+(1-1 / \sqrt{2}) \mathbb{1}\right)\right)$, and so in this sense it is sufficient to only consider equatorial measurements. On the other hand, it follows from Ref. [40] that for $p \geqslant 1 / 2$ any equatorial measurements on the noisy GHZ state (15) will produce local full correlators [41]. This implies that, no full-correlator inequality (for any number of measurements) is able to detect the nonlocality of state (15) for $N=3$ in the region $p \geqslant 1 / 2$. Notwithstanding, the nonlocality in this region is successfully detected by the $\mathrm{CHSH}$ method.

\section{Transversal dephasing}

We now analyze the case of the GHZ state (2) under dephasing along the transversal $X$ direction. The noisy state is now given by

$$
\rho_{N}^{x}=\sum_{\substack{k_{i}=0,1 \\ i=1, \ldots, N}}\left(1-\frac{p}{2}\right)^{N-k} \frac{p^{k}}{2} \Pi\left(\left|\mathrm{GHZ}_{N}^{\mathbf{k}}\right\rangle / \mathrm{GHZ}_{N}^{\mathbf{k}} \mid\right),
$$

with $\mathbf{k}=\left(k_{1}, k_{2} \ldots k_{N}\right), k_{i}=0$ or $1, k=\sum_{i=1, \ldots, N} k_{i}$, and where $\Pi\left(\left|\mathrm{GHZ}_{N}^{\mathbf{k}}\right\rangle\left\langle\mathrm{GHZ}_{N}^{\mathbf{k}}\right|\right)$ stands for the sum of all the $\left(\begin{array}{c}N \\ k\end{array}\right)$ different permutations of $\left|\mathrm{GHZ}_{N}^{\mathbf{k}}\right\rangle \doteq X_{1}^{k_{1}} \otimes \cdots \otimes X_{N}^{k_{N}}\left|\mathrm{GHZ}_{N}\right\rangle$ with $X_{i}^{0}=\mathbb{1}$. The noisy state (17) does not have a simple form as (15), and the optimal measurements for the MK inequality depend now on both $N$ and $p$. Analytical expressions for the MK violation and the corresponding noise threshold as functions of $N$ and $p$ are not available. However, using the multipartite CHSH method, a straightforward analysis is possible.

Applying the projector $(|+\rangle\langle+|)^{\otimes N-2}$, with support on all but qubits $i$ and $j$, to (17) results in the two-qubit state $\rho_{2}^{x}=\left(\left(1-\frac{p}{2}\right)^{2}+\left(\frac{p}{2}\right)^{2}\right)\left|\mathrm{GHZ}_{2}\right\rangle\left\langle\mathrm{GHZ}_{2}\right|+2\left(1-\frac{p}{2}\right)$ $\left(\frac{p}{2}\right)\left(X_{i} \otimes \mathbb{1}_{j}\right)\left|\mathrm{GHZ}_{2}\right\rangle\left\langle\mathrm{GHZ}_{2}\right|\left(X_{i} \otimes \mathbb{1}_{j}\right)$. For this state, one finds $\mathcal{M}_{\mathrm{CHSH}}^{x}=\sqrt{1+(1-p)^{4}}$. The noise threshold obtained is again $p_{c}^{x}=1$, independently of $N$, which reflects the entanglement robustness of GHZ states under transversal local dephasing $[42,43]$.

\section{General Pauli channels}

An analytical expression for the GHZ state under the general Pauli channel (5) can be obtained. Even though the evolved state is GHZ diagonal, analytical expressions for the MK violation are again not available. However, the $\mathrm{CHSH}$ method offers again a readily calculable bound. One obtains then $\mathcal{M}_{\mathrm{CHSH}}=$ $\sqrt{\left(p_{0}+p_{1}-p_{2}-p_{3}\right)^{2 n}+\left(p_{0}-p_{1}-p_{2}+p_{3}\right)^{4}}$. As a particular interesting case, we analyze approximate transversal local dephasing defined by $\alpha_{1}=1-\epsilon, \alpha_{2}=\epsilon / 2$, and $\alpha_{3}=\epsilon / 2$. The parameter $\epsilon$ thus measures the deviation of perfect transversal dephasing. In this case, $\mathcal{M}_{\mathrm{CHSH}}=$ $\sqrt{(1-p \epsilon)^{2} N+(1-p(1-\epsilon / 2))^{4}}$, which, for small values of $p$, can be approximated as $\mathcal{M}_{\mathrm{CHSH}} \approx \sqrt{1+(1-p)^{2 N \epsilon}}$, yielding an exponential decay with $N$, as with parallel dephasing, but with the decay rate reduced by a factor $\epsilon$, in a similar fashion to what happens with the entanglement in these noisy states $[42,43]$.

\section{Nonlocal content of noisy GHZ states}

To obtain a good lower bound for the local content we use the inequality (14). For GHZ states (15) under parallel local dephasing, $\rho_{1}$ and $\rho_{2}$ are given by

$\rho_{1,2}=(1-p)^{N}\left|\mathrm{GHZ}_{2}^{ \pm}\right\rangle\left\langle\mathrm{GHZ}_{2}^{ \pm}\right|+\left(1-(1-p)^{N}\right) \tilde{\rho}_{2}^{z}$,

with $\left|\mathrm{GHZ}_{2}^{ \pm}\right\rangle=(1 \sqrt{2})|00\rangle \pm|11\rangle$. In this case $p_{1}=p_{2}=1 / 2$. Choosing $A_{0}=Z, A_{1}=X, B_{0}=\cos (\theta) Z+\sin (\theta) X$, and $B_{1}=\cos (\theta) Z-\sin (\theta) X$ we find the left-hand side of (12) and (13) to be equal to $\cos (\theta)+\sin (\theta)(1-p)^{N}$. It is a simple calculation to show that choosing $\theta=\mathrm{s}^{-1}\left(\sqrt{1+(1-p)^{2 N}}\right)$ the latter value equals $\mathcal{M}_{\mathrm{CHSH}}^{z}$.

So for the GHZ state under parallel dephasing the $\mathrm{CHSH}$ method leads to the following lower bound on the nonlocal content,

$$
\tilde{p}_{\mathrm{NL}} \geqslant \sqrt{1+(1-p)^{2 N}}-1 .
$$

In Fig. 1, this bound is compared with the lower bound obtained in Ref. [38] through the MK inequality and with a numerical estimate, for $N=3$. To obtain the numerical estimate we first note that, for $N=3$ and two dichotomic measurements per party, all the facets of the local polytope are known, the socalled Sliwa inequalities [16]. We have optimized the violation of Sliwa inequalities over all possible projective measurements and using (8) obtained the optimal lower bound on $\tilde{p}_{\mathrm{NL}}$. As

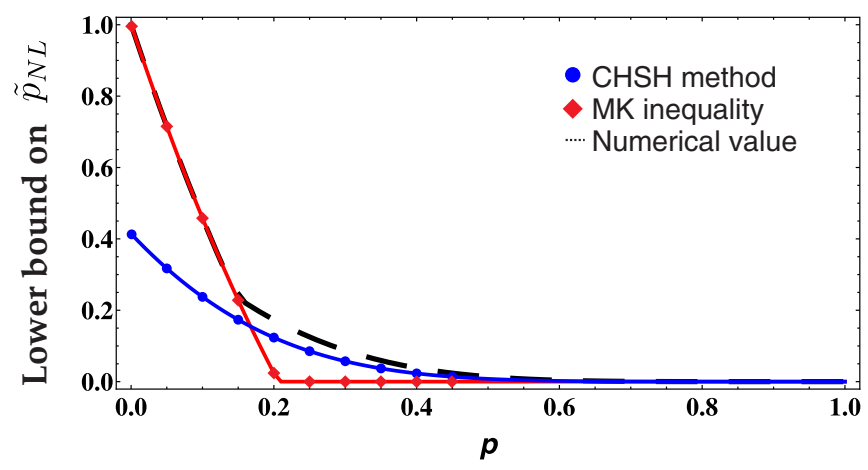

FIG. 1. (Color online) Lower bounds on the local content of the GHZ state under parallel dephasing, for $N=3$. (Red) The bound obtained from the MK inequality [38]; (blue) the new bound (19) from the CHSH method; and (black dashed) the value obtained through a numerical optimization described in the main text. For $p>0.18$, the nonlocal content is better described by the bound from the $\mathrm{CHSH}$ method. 


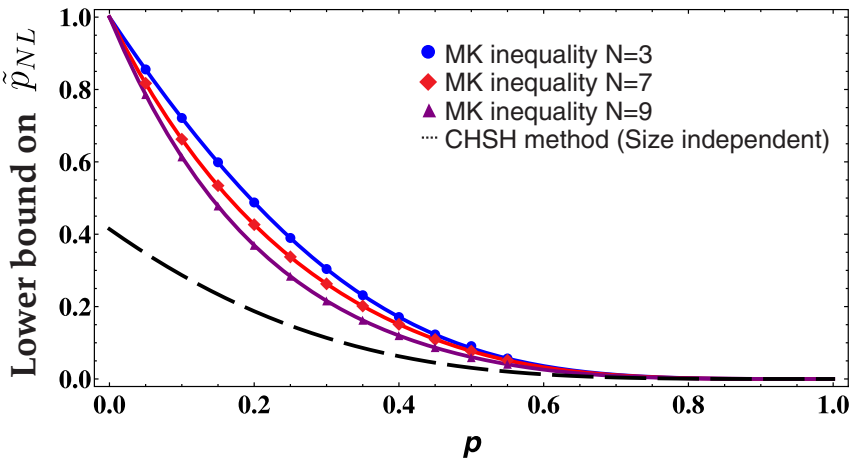

FIG. 2. (Color online) Lower bounds on the local content of the GHZ state under transversal dephasing. (Blue) Lower bound from the MK inequality of $N=3$; (red) idem for $N=7$; (purple) idem for $N=9$; (black dashed) lower bound (20) from the CHSH method. The bounds from the MK inequality were obtained through numerical optimization over all possible projective measurements. The $\mathrm{CHSH}$ bound is analytical and independent of $N$.

can be seen in Fig. 1, for most of the dynamics, bound (19) is tighter than the bound given by the MK inequality.

A similar calculation shows that for GHZ states (17) under transversal local dephasing, the $\mathrm{CHSH}$ method gives

$$
\tilde{p}_{\mathrm{NL}} \geqslant \sqrt{1+(1-p)^{4}}-1 \text {. }
$$

An analytical expression for the optimal MK violation is not available, as mentioned before. We numerically optimize the MK violation and so derive a numerical lower bound in the nonlocal content, plotted in Fig. 2 together with bound (20). The numerical MK bound is tighter, but the required optimization soon becomes unfeasible as $N$ grows. Bound (20), in contrast, is analytical and does not depend on $N$.

\section{B. Noisy $W$ states}

Let us now consider the nonlocality of the noisy $W$ state (21). We will consider dephasing along the $z$ direction in each of its qubits, which produces the state [44],

$$
\rho_{W_{N}}=\frac{1}{N}\left(1-p^{\prime}\right) \Pi(|00 \ldots 01\rangle\langle 00 \ldots 01|)+p^{\prime}|W\rangle\langle W|,
$$

with $p^{\prime}=(1-p)^{2}$ and $\Pi($.$) stands for all the permutations.$ The measurement outcome corresponding to the projector $|0\rangle\left\langle\left. 0\right|^{\otimes N-2}\right.$ (that occurs with probability $p=2 / N$ ), produces a two-qubit entangled state of the form $\rho_{W}^{2}$ for which the $\mathrm{CHSH}$ violation is $\mathcal{M}_{\mathrm{CHSH}}=\sqrt{1+(1-p)^{4}}$. So we recover the result in Ref. [19] that the dephased $W$ state is nonlocal through all the noisy dynamics, that is, $p_{c}=1$.

Once again we can use the multipartite $\mathrm{CHSH}$ method to provide a lower bound to the nonlocal content of this state. However, in this case the project states associated with other measurement outcomes other than $|0\rangle\left\langle\left. 0\right|^{\otimes N-2}\right.$ are not local unitarily equivalent to $\rho_{W}^{2}$. Actually they turn out to be separable and given by $|0\rangle\left\langle\left. 0\right|^{\otimes N-2}\right.$. Because of that, we must use expression (11) to calculate the lower bound to the local content, which renders

$$
\tilde{p}_{\mathrm{NL}} \geqslant\left(2 \sqrt{1+(1-p)^{4}}-2\right) / N .
$$

This bound provides a better estimate for the nonlocal content of (21) when compared to the one that can be obtained from the Bell inequality used in Ref. [19]. There the inequality used has a nonsignaling bound that increases exponentially with the number $N$ of qubits, while the violation given by the $W$ state is approximately independent of $N$. This makes the lower bound decay exponentially while our bound only decays linearly with $N$.

\section{Noisy graph states}

As the last application of the multipartite CHSH method, we study the nonlocality properties of graph states (4) subject to Pauli channels. In Ref. [45], multipartite Bell inequalities specially tailored to detect the nonlocality of graph states have been introduced. For some of these states, these inequalities are violated exponentially in $N$. Moreover, the violation, for any graph state under any Pauli channel, can be analytically expressed in a compact closed form (see Appendix).

For instance, for graph states under parallel local dephasing, their violation always decreases exponentially fast in $N$, which implies that the associated lower bound on the local content also decreases exponentially with $N$. Nevertheless, it is known that the entanglement in graph states is robust against local noise $[46,47]$. With the CHSH method, one easily shows that such entanglement robustness is also reflected in the nonlocality robustness.

As an illustration consider a star graph consisting of $N-1$ disconnected qubits connected to one central qubit. One simply projects all but the central qubit and one of the mutually disconnected qubits in the computational basis. Because the projection commutes with the dephasing channel, the two possible final states $\rho_{1}$ and $\rho_{2}$ (with $p_{1}=p_{2}=1 / 2$ ) are also a two-qubit graph state under parallel local dephasing (up to local unitaries). The left-hand side of (12) and (13) are equal to $\mathcal{M}_{\mathrm{CHSH}}=(1-p) \sqrt{2}$. This implies $p_{c}=1-\sqrt{1 / 2}$ and the robust (size-independent) bound

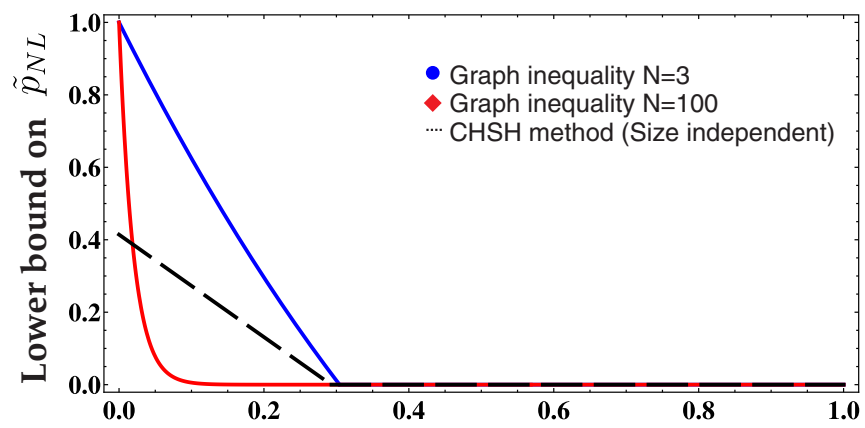

FIG. 3. (Color online) Lower bounds on the nonlocal content for star graph state of $N$ qubits and $|I|=N-1$ (see Appendix for details) and under parallel local dephasing. (Red and blue) The bounds obtained from the inequalities of Ref. [45], for $N=3$ and $N=100$, respectively; (black dashed) the new bound given by the $\mathrm{CHSH}$ method. The CHSH bound is size independent and offers an exponentially tighter estimate as $N$ increases. 
$\tilde{p}_{\mathrm{NL}} \geqslant \max [0,(1-p) \sqrt{2}-1]$. For large $N$ and $p<p_{c}$, this bound is exponentially tighter than that obtained from the Bell inequalities of [45], as shown in Fig. 3.

\section{SUMMARY}

In this work we have used the CHSH inequality in the multipartite scenario, and showed its usefulness to detect the nonlocality of noisy multipartite states. The method consists of locally projecting the multipartite state into a nonlocal two-qubit state that violates the CHSH inequality. We have shown examples of states for which the nonlocality cannot be detected by the WWWZB inequalities consisting only of full correlators (actually, any full-correlator inequality if $N=3$ ), but can be detected by the present method. The multipartite CHSH method works well also in situations where it is difficult to analytically find optimal Bell inequality violations, as for GHZ states undergoing transversal dephasing. Furthermore, the method can be easily applied to obtain tight lower bounds to the nonlocal content of correlations.

We believe these findings should contribute to the detection of nonlocality for noisy multipartite states. In particular, the present method seems to be the simplest one to experimentally detect nonlocality in multipartite states.

\section{ACKNOWLEDGMENTS}

We thank N. Brunner for motivating us to write this paper and the Benasque Center for Science for hospitality during the Quantum Information Workshop 2013. We also thank the referee for his/her useful comments. This work was supported by the Excellence Initiative of the German Federal and State Governments (Grant No. ZUK 43), the EU project SIQS, and the National Institute of Science and Technology for Quantum Information, the National Research Foundation, and the Ministry of Education of Singapore. L.A. acknowledges support from the EU under Marie Curie IEF Grant No. 299141.

\section{APPENDIX: GRAPH-STATE BELL INEQUALITIES UNDER PAULI CHANNELS}

Given a vertex $i$ of a graph state $\left|G_{0}\right\rangle$ of $N$ qubits and a subset of its neighbors $I \subseteq \mathcal{N}(i)$, such that none of the vertices in $I$ are connected by an edge, the Bell operator $\mathcal{B}(i, I)=$ $K_{i} \prod_{j \in I}\left(1+K_{j}\right)\left(K_{i}=X_{i} \prod_{j \in \mathcal{N}_{i}} Z_{j}\right.$ are the generators of the graph, that is, $\left.K_{i}\left|G_{\mathbf{0}}\right\rangle=\left|G_{\mathbf{0}}\right\rangle\right)$ defines a Bell inequality given by [45]

$$
|\langle\mathcal{B}\rangle|=|\langle\mathcal{B}(i, I)\rangle| \leqslant L(|I|+1),
$$

with a classical bound given by $L(m)=2^{(m-1) / 2}$ for $m$ odd, and $L(m)=2^{m / 2}$ for $m$ even. The inequality is maximally violated by the graph $\left|G_{0}\right\rangle$ with $\langle\mathcal{B}(i, I)\rangle=2^{|I|}$.

Under the action of a Pauli map, the graph state will turn into a graph diagonal mixed state $\rho_{G}=\sum p_{\mu}\left|G_{\mu}\right\rangle\left\langle G_{\mu}\right|$, with $\left|G_{\mu}\right\rangle=Z^{\mu_{1}} \otimes Z^{\mu_{2}} \cdots \otimes Z^{\mu_{N}}\left|G_{\mathbf{0}}\right\rangle$, where $\mu=\left(\mu_{1}, \ldots, \mu_{N}\right)$ is a multi-index, $\mu_{j}$ can assume values 0 or 1 , and the weights $p_{\mu}$ depend on the exact form of the Pauli map. The expectation value of the Bell operator $\mathcal{B}(i, I)$ on this state is given by

$$
\begin{aligned}
\langle\mathcal{B}\rangle_{\rho_{G}} & =\left\langle\sum p_{\mu} K_{i} \prod_{j \in I}\left(1+K_{j}\right) \mid G_{\mu}\right\rangle\left\langle G_{\mu} \mid\right\rangle \\
& =\sum p_{\mu}(-1)^{\mu_{i}} \prod_{j \in I}\left(1+(-1)^{\mu_{j}}\right),
\end{aligned}
$$

where we have used that $K_{i}\left|G_{\mu}\right\rangle=(-1)^{\mu_{i}}\left|G_{\mu}\right\rangle$. From (A2) it follows that the only terms in the convex sum $\rho_{G}=$ $\sum p_{\mu}\left|G_{\mu}\right\rangle\left\langle G_{\mu}\right|$ contributing to the expectation of the Bell operator are $\mu^{0}=(0,0, \ldots, 0)$ and $\mu^{1}=(1,0, \ldots, 0)$, that is, $\langle\mathcal{B}\rangle_{\rho_{G}}=\left(p_{\mu^{0}}-p_{\mu^{1}}\right) 2^{|I|}$.

As a matter of fact, consider any graph state undergoing local dephasing. From (A2) we see that $\langle\mathcal{B}\rangle_{\rho_{G}}=(1-p)$ $(1-p / 2)^{N-1} 2^{|I|}$, which shows an exponential decay in the violation with $N$, that is also reflected in a exponential decay of the associated lower bound for the nonlocal content (see Fig. 3).
[1] J. S. Bell, Physics 1, 195 (1964).

[2] N. Brunner, D. Cavalcanti, S. Pironio, V. Scarani, and S. Wehner, arXiv:1303.2849.

[3] J.-D. Bancal, N. Gisin, Y.-C. Liang, and S. Pironio, Phys. Rev. Lett. 106, 250404 (2011).

[4] H. Buhrman, R. Cleve, S. Massar, and R. de Wolf, Rev. Mod. Phys. 82, 665 (2010).

[5] A. Acín, N. Brunner, N. Gisin, S. Massar, S. Pironio, and V. Scarani, Phys. Rev. Lett. 98, 230501 (2007).

[6] J. Barrett, L. Hardy, and A. Kent, Phys. Rev. Lett. 95, 010503 (2005).

[7] A. Acín, N. Gisin, and L. Masanes, Phys. Rev. Lett. 97, 120405 (2006).

[8] S. Pironio, A. Acín, S. Massar, A. B. de la Giroday, D. N. Matsukevich, P. Maunz, S. Olmschenk, D. Hayes, L. Luo, T. A. Manning, and C. Monroe, Nature (London) 464, 1021 (2010).

[9] R. Colbeck and A. Kent, J. Phys. A: Math. Theor. 44, 095305 (2011).

[10] R. Colbeck and R. Renner, Nat. Phys. 8, 450 (2012).
[11] R. Gallego, Ll. Masanes, G. de la Torre, C. Dhara, L. Aolita, and A. Acín, Nat. Commun. 4, 2654 (2013).

[12] R. Ramanathan, F. G. S. L. Brandao, A. Grudka, K. Horodecki, M. Horodecki, and P. Horodecki, arXiv:1308.4635.

[13] J. F. Clauser, M. A. Horne, A. Shimony, and R. A. Holt, Phys. Rev. Lett. 23, 880 (1969).

[14] A. Fine, Phys. Rev. Lett. 48, 291 (1982).

[15] R. Horodecki, P. Horodecki, and M. Horodecki, Phys. Lett. A 200, 340 (1995).

[16] C. Sliwa, Phys. Lett. A 317, 165 (2003).

[17] I. Pitowsky, Quantum Probability-Quantum Logic, Lecture Notes in Physics (Springer-Verlag, Berlin, 1989).

[18] I. Pitowsky, Math. Program. 50, 395 (1991).

[19] W. Laskowski, T. Paterek, C. Brukner, and M. Zukowski, Phys. Rev. A 81, 042101 (2010).

[20] A. C. Elitzur, S. Popescu, and D. Rohrlich, Phys. Lett. A 162, 25 (1992).

[21] S. Popescu and D. Rohrlich, Phys. Lett. A 166, 293 (1992). 
[22] D. M. Greenberger, M. A. Horne, and A. Zeilinger, in Bell's Theorem, Quantum Theory, and Conceptions of the Universe, edited by M. Kafatos (Kluwer, Dordrecht, 1989).

[23] W. Dür, G. Vidal, and J. I. Cirac, Phys. Rev. A 62, 062314 (2000).

[24] M. Hein, J. Eisert, and H. J. Briegel, Phys. Rev. A 69, 062311 (2004).

[25] M. Hein, W. Dür, J. Eisert, R. Raußendorf, M. Van den Nest, and H. Briegel, in International School of Physics Enrico Fermi (Varenna, Italy), Quantum Computers, Algorithms and Chaos, edited by P. Zoller, G. Casati, D. Shepelyansky, and G. Benenti, Vol. 162 (International School of Physics Enrico Fermi, Varenna, 2006).

[26] J. Barrett, A. Kent, and S. Pironio, Phys. Rev. Lett. 97, 170409 (2006).

[27] D. Cavalcanti, M. L. Almeida, V. Scarani, and A. Acín, Nat. Commun. 2, 184 (2011).

[28] D. Cavalcanti, R. Rabelo, and V. Scarani, Phys. Rev. Lett. 108, 040402 (2012).

[29] W. Kłobus, W. Laskowski, M. Markiewicz, and A. Grudka, Phys. Rev. A 86, 020302 (2012).

[30] L. Aolita, R. Chaves, D. Cavalcanti, A. Acín, and L. Davidovich, Phys. Rev. Lett. 100, 080501 (2008).

[31] L. Aolita, D. Cavalcanti, A. Acín, A. Salles, M. Tiersch, A. Buchleitner, and F. de Melo, Phys. Rev. A 79, 032322 (2009).

[32] R. F. Werner and M. M. Wolf, Phys. Rev. A 64, 032112 (2001).

[33] H. Weinfurter and M. Zukowski, Phys. Rev. A 64, 010102 (2001).

[34] M. Zukowski and C. Brukner, Phys. Rev. Lett. 88, 210401 (2002).

[35] N. D. Mermin, Phys. Rev. Lett. 65, 1838 (1990).

[36] A. V. Belinskii and D. N. Klyshko, Phys. Usp. 36, 653 (1993).

[37] V. Scarani and N. Gisin, J. Phys. A: Math. Gen. 34, 6043 (2001).

[38] R. Chaves, D. Cavalcanti, L. Aolita, and A. Acín, Phys. Rev. A 86, 012108 (2012).
[39] In general we are interested in the maximal violation of $\mathrm{CHSH}$ inequality that can be obtained by the projection of $N-2$ qubits. Note that the projected two-qubit state can, in principle, be explicitly written as a function of the $2(N-2)$ angles and the $N-2$ dichotomic parameters describing, respectively, the projectors and its outcomes. One can then maximize the $\mathrm{CHSH}$ violations (via the criterion in Ref. [15]) optimizing over these variables. For example, for GHZ states under parallel and transversal dephasing we have numerically observed (up to $N=5$ ) that the projections in the $X$ basis are optimal. We have considered this projective measurement for arbitrary $N$, which provides a lower bound on the critical noise strength $p_{c}$ and the nonlocal weight $\tilde{p}_{\mathrm{NL}}$.

[40] C. Branciard and N. Gisin, Phys. Rev. Lett. 107, 020401 (2011).

[41] This can be seen from the results in Ref. [40] as follows. Branciard and Gisin showed a way of simulating the correlations of equatorial von Neuman measurements on the tripartite GHZ state using three bits of communication. The same result can be used to show a local model for the full correlators of the form $(1-p)^{3}\left\langle\mathrm{GHZ}_{3}\left|\sigma_{i} \otimes \sigma_{j} \otimes \sigma_{k}\right| \mathrm{GHZ}_{3}\right\rangle$ for $i, j, k=1,2$ and $p \geqslant 1 / 2$ (see section Detection loophole in Ref. [40]). These are the same full correlations obtained from equatorial von Neumann measurements on the state $(15)(N=3)$ with $p \geqslant 1 / 2$.

[42] R. Chaves, L. Aolita, and A. Acín, Phys. Rev. A 86, 020301 (2012).

[43] R. Chaves, J. B. Brask, M. Markiewicz, J. Kołodyński, and A. Acin, Phys. Rev. Lett. 111, 120401 (2013).

[44] R. Chaves and L. Davidovich, Phys. Rev. A 82, 052308 (2010).

[45] G. Tóth, O. Gühne, and H. J. Briegel, Phys. Rev. A 73, 022303 (2006).

[46] D. Cavalcanti, R. Chaves, L. Aolita, L. Davidovich, and A. Acín, Phys. Rev. Lett. 103, 030502 (2009).

[47] L. Aolita, D. Cavalcanti, R. Chaves, C. Dhara, L. Davidovich, and A. Acin, Phys. Rev. A 82, 032317 (2010). 